\title{
Flavobacterium meningosepticum in the hospital environment
}

\author{
MARGARET M. COYLE-GILCHRIST, PATRICIA CREWE, AND G. ROBERTS \\ From the Department of Pathology, Maelor General Hospital, Wrexham
}

SYNOPSIS Ten strains of Flavobacterium meningosepticum were isolated from routine clinical material during a period of two years. Of a number of antibiotics tested, clindamycin was the only one to which they were fully sensitive. The organism was found in chlorhexidine gluconate (Hibitane) solutions in use on the wards for the storage of thermometers and for routine disinfection.

Flavobacterium meningosepticum was isolated from 10 patients between July 1973 and July 1975. It was isolated from cultures of blood and of cerebrospinal fluid from one patient, from blood cultures in five others, and from one abdominal wound swab, one skin swab, one chest drain catheter, and one intracardiac catheter. Lapage and Owen (1973) describe the first case in England from an infant who was probably infected in Bangladesh, but no further cases have been recorded. In view of the extreme resistance to antibiotics and rarity of the organism, environmental studies were carried out in an attempt to trace its source.

\section{Material and methods}

The Flavobacterium cultures were originally identified by the Computer Trials Laboratory at Colindale and then, following the recommended techniques, at our own laboratory. The biochemical tests were carried out according to the methods of Cowan and Steel (1974).

Environmental cultures were carried out using a moist swab for surfaces and the in-use test of Kelsey and Maurer (1966) for disinfectants.

Sensitivity testing was carried out by spot inoculating a $10^{-4}$ dilution of an overnight broth culture incubated at room temperature onto Wellcotest agar containing doubling dilutions of antibiotics, incubation being at room temperature.

\section{Results}

IDENTIFICATION

The organisms were Gram-negative non-motile rods.

Received for publication 24 February 1976
After 24 hours' incubation at $37^{\circ} \mathrm{C}$ the colonies were $0.5-1 \mathrm{~mm}$ in diameter. A few strains took $48 \mathrm{c}$ hours for growth to become visible. No pigment production was seen. All the strains gave positive results in the following tests: oxidase, catalase, growth on MacConkey agar, ONPG, DNase, aesculin hydrolysis, and gelatin liquefaction; glucose $\vec{\theta}$ was utilized by oxidation in the O-F test. Negatio oे results were obtained in the following tests: indole $\mathrm{H}_{2} \mathrm{~S}$ paper, peptone water sugars, lysine and ornithine decarboxylase, arginine dihydrolase, PPA, malonate, gluconate, Simmons citrate, and starch hydrolysis. Growth did not take place at $5^{\circ} \mathrm{C}$ or $\stackrel{\mathrm{D}}{\mathrm{D}}$ $42^{\circ} \mathrm{C}$.

The important distinguishing features were $\frac{\vec{O}}{3}$ improved growth at room temperature, ONPG, oxidase, gelatin, and DNase positive reactions and negative indole, $\mathrm{H}_{2} \mathrm{~S}$, and starch hydrolysis tests, the latter distinguishing it from Flavobacterium group IIB, which hydrolyses starch.

The first few cultures of $\boldsymbol{F}$. meningosepticum were typed serologically at the Computer Trials Laboratory and were found to belong to group F.

\section{SENSITIVITY}

Clindamycin was the only antibiotic tested with a minimum inhibitory concentration within the fully sensitive range (table).

CLINICAL DETAILS

One patient appears to have been infected with $F$. $O$ meningosepticum. This was a 6-week-old baby admitted with a strangulated inguinal hernia and $\stackrel{C}{\mathscr{D}}$ clinical signs of septicaemia. F. meningosepticum was $\stackrel{\oplus}{+}$ isolated from blood cultures taken on admission and also from cerebrospinal fluid collected the following day. The identity of the organism was not known but 824 


\begin{tabular}{lc}
\hline Antibiotic & $M I C(\mu \mathrm{g} / \mathrm{ml})$ \\
\hline Clindamycin & 4 \\
Tetracycline & 16 \\
Erythromycin & 64 \\
Carbenicillin & 64 \\
Gentamycin & over 128 \\
Tobramycin & over 128 \\
Ampicillin & over 128 \\
Cephalosporins & over 128 \\
Kanamycin & over 128 \\
Colomycin & over 128 \\
Trimethoprim & over 128 \\
Sulphamethoxazole & over 128 \\
\hline
\end{tabular}

Table Sensitivity of 10 strains of Flavobacterium meningosepticum

it was thought to belong to the Pseudomonas group. Sensitivity to clindamycin was not tested at this time. The organism was moderately sensitive to carbenicillin and the child recovered on this treatment. The hernia was reduced surgically without the need for resection of the bowel.

It is almost certain that none of the other patients was infected and that the organism came from contaminated solutions used for disinfection of the skin. This certainly occurred in the next to last patient in the series. He was a 58-year-old man diagnosed clinically and by the isolation from blood cultures of a non-haemolytic streptococcus as a subacute bacterial endocarditis in December 1974. He made an uneventful recovery and was discharged from hospital two months later.

While attending outpatients in May 1975 he complained of having had several transient attacks of diplopia. He was apyrexial and had no other symptoms, and it was thought unlikely that he had suffered a recrudescence of the infection, but blood cultures were collected to exclude this. The growth of $F$. meningosepticum from these cultures prompted the investigation of the Hibitane solutions used to disinfect this patient's skin. When this, too, yielded $F$. meningosepticum the investigation of the disinfectant solutions was carried out.

Details of the remaining patients who yielded $F$. meningosepticum are as follows:

A 2-year-old child was admitted with fever and lymphadenopathy which was found to be due to infection with adenovirus type 7 .

A 6-year-old child was admitted with febrile convulsions; cerebrospinal fluid was normal and sterile.

Another 6-year-old was admitted with an abscess of the foot and a thrombophlebitis and lymphangitis of the leg. Culture of the abscess yielded Staphylococcus aureus.

An 82-year-old woman had cirrhosis of the liver and recurrent attacks of pyrexia. Repeat blood cultures were sterile.
A 47-year-old woman was found at laparotomy to have acute pancreatitis. Swabs from the wound drain grew $F$. meningosepticum on one occasion only.

A 31-year-old woman had had discharge from a nipple which had produced eczema of the surrounding skin and grew $F$. meningosepticum. A subsequent swab produced Staph. aureus.

Culture of the catheter removed from the chest of a 58-year-old woman who was a chronic bronchitic and had suffered a pneumothorax yielded $F$. meningosepticum. The patient had no signs of infection of the pleural cavity 0 - the drain site.

A 13-year-old boy was operated on for intestinal obstruction which necessitated the resection of a portion of terminal ileum. A catheter was inserted into the right atrium to measure the central venous pressure. On removal the catheter tip was sent for culture and $F$. meningosepticum was grown. This organism was not isolated on subsequent blood cultures.

\section{SOURCE}

$F$. meningosepticum was found in most of the thermometer storage solutions containing a 1 in 4000 aqueous solution of chlorhexidine (Hibitane) on all 26 wards, and in all the aqueous chlorhexidine solutions of 1 in 1000 and 1 in 5000 used as ward disinfectants. In most cases more than 1000 organisms per $\mathrm{cm}^{3}$ were isolated. The organism was present in two separate hospitals, both with their own independent pharmacies. Extensive testing of water supplies and stock solutions of chlorhexidine was carried out at the pharmacies but no Flavobacterium was found. None was present in the Hibiscrub or the $0.5 \%$ chlorhexidine in spirit. Further investigations revealed that nail brushes and some instruments were stored in contaminated solutions of chlorhexidine.

\section{Discussion}

There have been many instances of contamination of disinfectants with water-borne organisms (Linton and George, 1966; Bassett et al, 1970; Bassett, 1971; Maurer, 1974). Flavobacterium group IIB was found in ice used to cool syringes which were to be used to obtain arterial specimens for blood gas determination in Maryland, USA (Stamm et al, 1975).

The primary source of $F$. meningosepticum in the present investigation was never found, but it may have been the water, the topping up of the ward bottles ensuring the persistence of the organism. The probable source of infection of the patients was the contaminated chlorhexidine solutions used for storing thermometers, nail brushes, and some instruments, and also for cleansing the skin. It was 
noticeable that $F$. meningosepticum was not found in the Premature Baby Unit, where temperatures are taken under the armpit.

\section{Conclusion}

The presence of $F$. meningosepticum in the chlorhexidine solutions serves to highlight the dangerous practice of storing thermometers and instruments in disinfectants and topping up stock solutions of disinfectants. Thermometers should be stored dry and disinfectants issued in disposable or autoclavable containers.

We thank Dr. S. P. Lapage and staff of the Computer Trials Laboratory, Colindale for identifying the original strains of Flavobacterium meningosepticum, and Mrs. E. L. Williams for typing the manuscript.

\section{References}

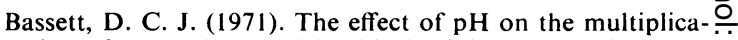
tion of a pseudomonad in chlorhexidine and cetrimide. $J . \vec{\Rightarrow}$ clin. P:sth., 24, 708-711.

Bassett, D. C. J., Stokes, K. J., and Thomas, W. R. G. (1970). Wound infection with Pseudomonas multivorans, a waterborne contaminant of disinfection solutions. Lancet, 1 1188-1191.

Cowan, S. T. and Steel, K. J. (1974). Manual for the Identification of Medical Bacteria, 2nd edition. Cambridge University
Press, London.

Kelsey, J. C. and Maurer, I. M. (1966). An in-use test for $\vec{O}$ hospital disinfectants. Mth Bull. Minist. Hlth Lab. Serv., 25, $180-184$.

Lapage, S. P. and Owen, R. J. (1973). Flavobacterium $\vec{\sigma}^{-}$ meningosepticum from cases of meningitis in Botswana

Linton, K. B. and George, E. (1966). Inactivation of chlor- if hexidine ('Hibitane') by bark corks. Lancet, 1, 1353-1355.

Maurer, I. M. (1974). Hospital Hygiene. Arnold, London.

Stamm, W. E., Colella, J. J., Anderson, R. L., and Dixon, $\stackrel{\infty}{N}$ R. E. (1975). Indwelling arterial catheters as a source of nosocomial bacteremia. New Engl.J. Med., 292, 1099-1102. 은 